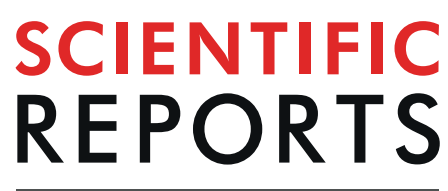

natureresearch

\title{
Manually curated genome-scale reconstruction of the metabolic network of Bacillus megaterium DSM319
}

\author{
Javad Aminian-Dehkordi $\mathbb{1}^{1}$, Seyyed Mohammad Mousavi ${ }^{1 *}$, Arezou Jafari ${ }^{2}$, Ivan Mijakovic ${ }^{3,4}$ \\ \& Sayed-Amir Marashi $\mathbb{D}^{5 *}$
}

Bacillus megaterium is a microorganism widely used in industrial biotechnology for production of enzymes and recombinant proteins, as well as in bioleaching processes. Precise understanding of its metabolism is essential for designing engineering strategies to further optimize $B$. megaterium for biotechnology applications. Here, we present a genome-scale metabolic model for $B$. megaterium DSM319, iJA1121, which is a result of a metabolic network reconciliation process. The model includes 1709 reactions, 1349 metabolites, and 1121 genes. Based on multiple-genome alignments and available genome-scale metabolic models for other Bacillus species, we constructed a draft network using an automated approach followed by manual curation. The refinements were performed using a gap-filling process. Constraint-based modeling was used to scrutinize network features. Phenotyping assays were performed in order to validate the growth behavior of the model using different substrates. To verify the model accuracy, experimental data reported in the literature (growth behavior patterns, metabolite production capabilities, metabolic flux analysis using ${ }^{13} \mathrm{C}$ glucose and formaldehyde inhibitory effect) were confronted with model predictions. This indicated a very good agreement between in silico results and experimental data. For example, our in silico study of fatty acid biosynthesis and lipid accumulation in B. megaterium highlighted the importance of adopting appropriate carbon sources for fermentation purposes. We conclude that the genome-scale metabolic model iJA1121 represents a useful tool for systems analysis and furthers our understanding of the metabolism of $B$. megaterium.

In recent decades, research on Bacillus megaterium has gained momentum due to its versatile metabolic capabilities and physical properties favorable to biotechnology applications. This bacterium had already been commonly used in biochemical studies before the extensive popularity of Bacillus subtilis ${ }^{1,2}$. Large cell size and special physiochemical properties were the main incentives to use B. megaterium as a model to study cell structure, sporulation, and protein localization ${ }^{3,4}$. As a Gram-positive bacterium with an aerobic sporulation behavior, $B$. megaterium inhabits diverse environments, ranging from dried food to soil ${ }^{5}$. Its ability to grow on a variety of carbon sources has made it amenable for industrial applications ${ }^{6}$. Numerous strains of B. megaterium have been applied for production of various enzymes, such as penicillin amidase, amylase, amino acid dehydrogenase, and glucose dehydrogenase, as well as for production of recombinant proteins ${ }^{7-11}$. Moreover, it has been used as an alternative microorganism for production of vitamin $\mathrm{B}_{12}$, pyruvate, and shikimate ${ }^{12-14}$. Remarkably, B. megaterium can also be utilized as a cyanogenic bacterium in the bioleaching process, in order to mobilize precious metals from e-wastes ${ }^{15}$.

Among various strains of B. megaterium, B. megaterium DSM319 (B. m. DSM319 hereafter) stands out as being extensively investigated. B. $m$. DSM319 and its available derivatives are commonly used for production of recombinant proteins ${ }^{16-18}$ and metabolites ${ }^{19-22}$. The whole genome of $B . m$. DSM319 was sequenced by Eppinger et al. ${ }^{23}$, which provided a wealth of information on its genotype-phenotype relationships. However, genome data

${ }^{1}$ Biotechnology Group, Department of Chemical Engineering, Tarbiat Modares University, Tehran, Iran. ${ }^{2}$ Department of Chemical Engineering, Tarbiat Modares University, Tehran, Iran. ${ }^{3}$ Department of Biology and Biological Engineering, Chalmers University of Technology, Göteborg, Sweden. ${ }^{4}$ Novo Nordisk Foundation Center for Biosustainability, Technical University of Denmark, Lyngby, Denmark. ${ }^{5}$ Department of Biotechnology, College of Science, University of Tehran, Tehran, Iran. *email: mousavi_m@modares.ac.ir; marashi@ut.ac.ir 


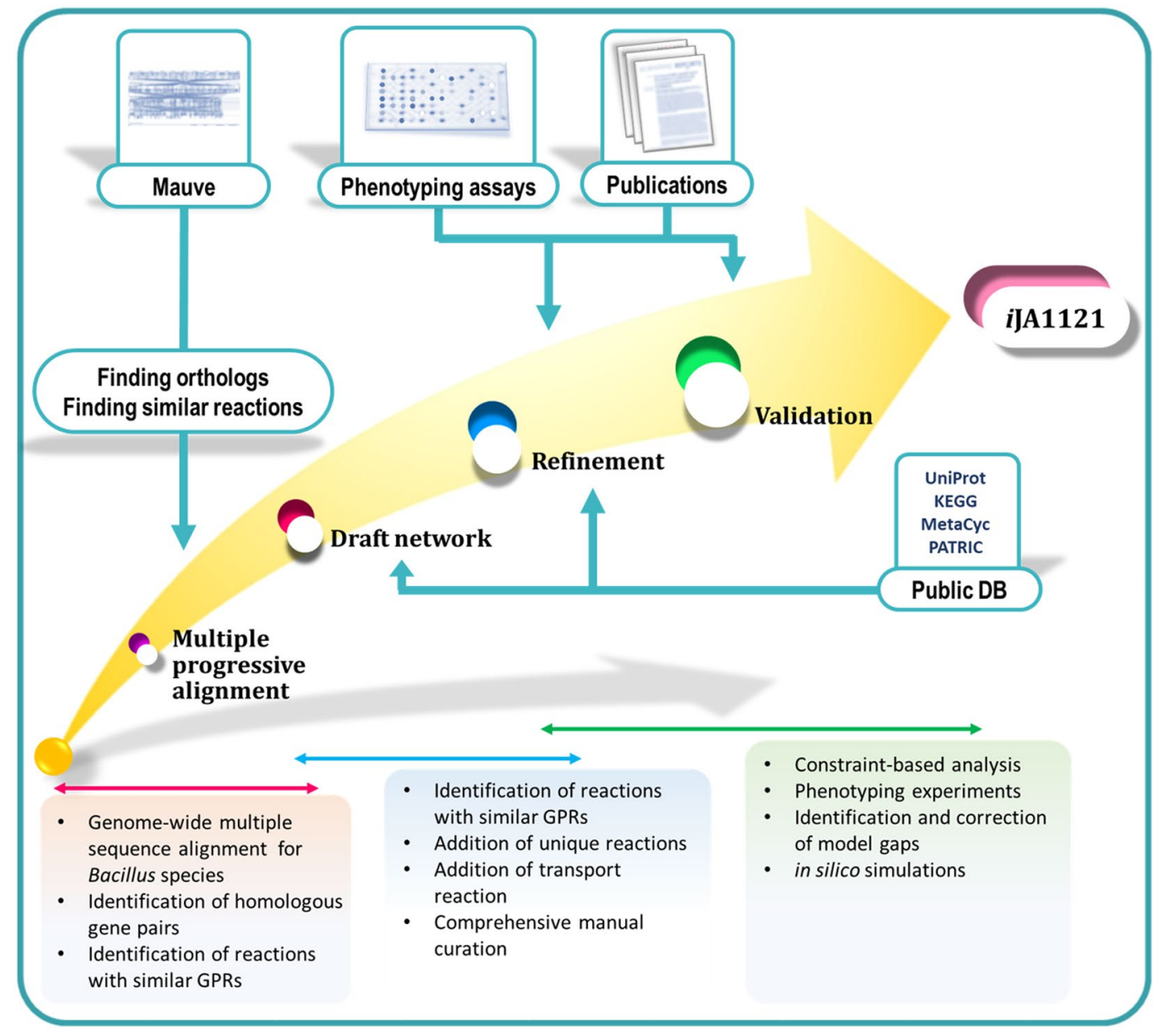

Figure 1. Schematic representation of the genome-scale metabolic network reconstruction procedure. Using Mauve, homologous gene pairs were detected to identify the reactions with similar gene-reaction associations in genome-scale metabolic models of Bacillus species. After the comprehensive manual curation, the draft model was validated and refined using phenotyping experiments and the experimental data reported in the literature.

alone was not sufficient for providing a holistic and comprehensive picture of the B. $m$. DSM319 metabolism. Therefore, there remains a knowledge gap that needs to be filled.

Genome-scale metabolic networks together with constraint-based modeling provide a computational framework that can predict physiological features of cells and organisms ${ }^{24}$. Genome-scale metabolic models (GEMs) are used to evaluate and determine the potential of industrial strains and explore their unknown metabolic capabilities $^{25}$. GEM-based predictions can be used for amelioration of culture medium composition, by identifying key metabolites that need to be added to increase the growth rate. They can also be used for predicting metabolite production fluxes ${ }^{26}$ and defining gene deletion strategies for metabolic engineering ${ }^{27}$.

A GEM for B. megaterium WSH002 (B. $\mathrm{m}$. WSH002 hereafter), iMZ1055, has been previously reported ${ }^{28}$. This model included some obsolete annotations and was shown not to be very successful in predicting fluxes ${ }^{29}$. In this study, a GEM has been developed for B. m. DSM319. This model reconciles $i$ MZ1055 and biochemical data specific to B. $m$. DSM319, taking into account other Bacillus metabolic models (including $i$ Bsu 1103 and $i$ Bsu1147 for B. subtilis, and $i$ WX1009 for B. licheniformis). Fig. 1 schematically represents the procedure of GEM reconstruction for B. $m$. DSM319. It should be emphasized that our model was able to accurately predict the metabolic functions and growth behavior of the strain. Model predictions were in agreement with the growth simulation results from Biolog phenotyping assays, as well as with several experimental data-sets reported in the literature.

\section{Results and Discussion}

Genome-scale reconstruction process. According to a reconciliation process, we used genome sequences of Bacillus species to identify potential reactions that should be present in the GEM of B. $m$. DSM319. Genome-wide multiple sequence alignment was performed prior to refinement and validation, in order to find orthologs. The detected homologous gene pairs were applied as references for identification of similar gene-protein-reaction (GPRs) associations to reconstruct the draft network. For this purpose, the homologous gene pairs of B. $m$. DSM319 and B. m. WSH002 were initially identified using Mauve ${ }^{30}$. Based on the results, 4526 coding sequence (CDS) homologous pairs were determined, which will be referred to as the "COM" genes (see Supplementary Information). Moreover, B. $m$. DSM319 had 692 CDSs with no obvious homolog in the B. $m$. 


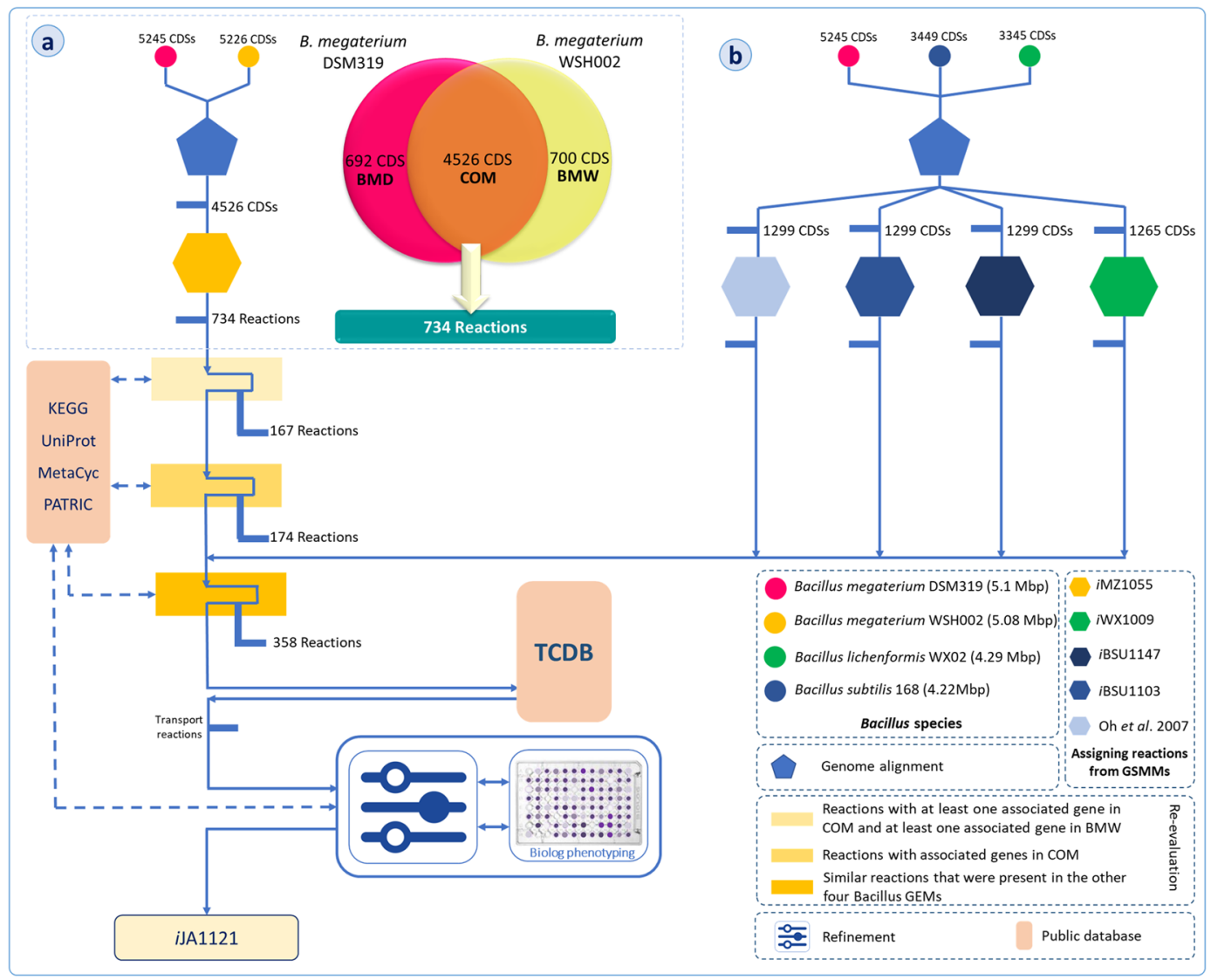

Figure 2. Schematic representation of the reconstruction process used for the genome-scale metabolic network of Bacillus megaterium DSM319. (a) Based on genome alignment, 734 reactions of $i$ MZ1055 were included in the reconstruction of the initial draft network. (b) The initial draft network was modified by performing several manual curation steps, utilization of Bacillus species GenBank genomes for multiple-alignment and further addition of relevant reactions to the model, and refining the model based on phenotyping data. Solid arrows represent the process direction and dotted lines relate to the operations that were performed based on the available data in public databases. "167 Reactions", "174 Reactions" and "358 Reactions" refer to "the reactions that had at least one associated gene in COM and at least one associated gene in BMW", "the reactions that were associated with some gene(s) of COM" and "the similar reactions that were present in the other four Bacillus GEMs", respectively (see Supplementary Information).

WSH002 genome. This set of genes will be called "BMD" genes (Fig. 2a). Those genes which are present in $B$. $m$. WSH002, but not in the B. $m$. DSM319 genome, will be called "BMW" genes. Further information about the reconstruction process is presented in Supplementary Information.

In the next step, the draft network was manually curated in order to find potential errors. Altogether, we resolved 314 errors, including the modification of GPR associations, EC numbers, metabolites, addition of several complex enzymes and isozymes, as well as modification of the relationships among genes using Boolean logical operators.

In order to find any potential missing reactions which are present in the other four Bacillus GEMs, we carried out a genome-wide multiple sequence alignment for B. megaterium, B. subtilis, and B. licheniformis. Based on the results (Fig. 2), 358 reactions were added to the draft network. Furthermore, the BMD genes, which are present in B.m. DSM319 only, together with their associated reactions which were obtained by KEGG API ${ }^{31}$, were added to the draft network.

Finally, the manual refinement step in the reconstruction of the B. $m$. DSM319 network was performed by examining the inconsistencies between the in silico predictions and experimental results (Biolog phenotyping and literature). Figure $2 \mathrm{~b}$ schematically represents the entire process of GEM reconstruction for B. $m$. DSM319.

Phenotyping assays. We performed Biolog phenotyping experiments to examine the capability of the draft network to predict growth behavior on different carbon sources. B. $m$. DSM319 was found to be capable of growing on 49 out of 69 different carbon sources based on triplicate independent experiments (Fig. 3). Growth on different carbon sources was simulated, as explained in the Materials and Methods section, using FBA ${ }^{32}$. Further refinements of the draft network were performed based on the observed phenotyping results and in silico predictions. For every carbon source, an independent simulation was run. Among 69 different carbon sources, 60 carbon sources matched transport reactions while nine were known as intracellular metabolites, with no transport reaction in the draft model. Therefore, in order to determine potential membrane transporters for the 9 suspected 


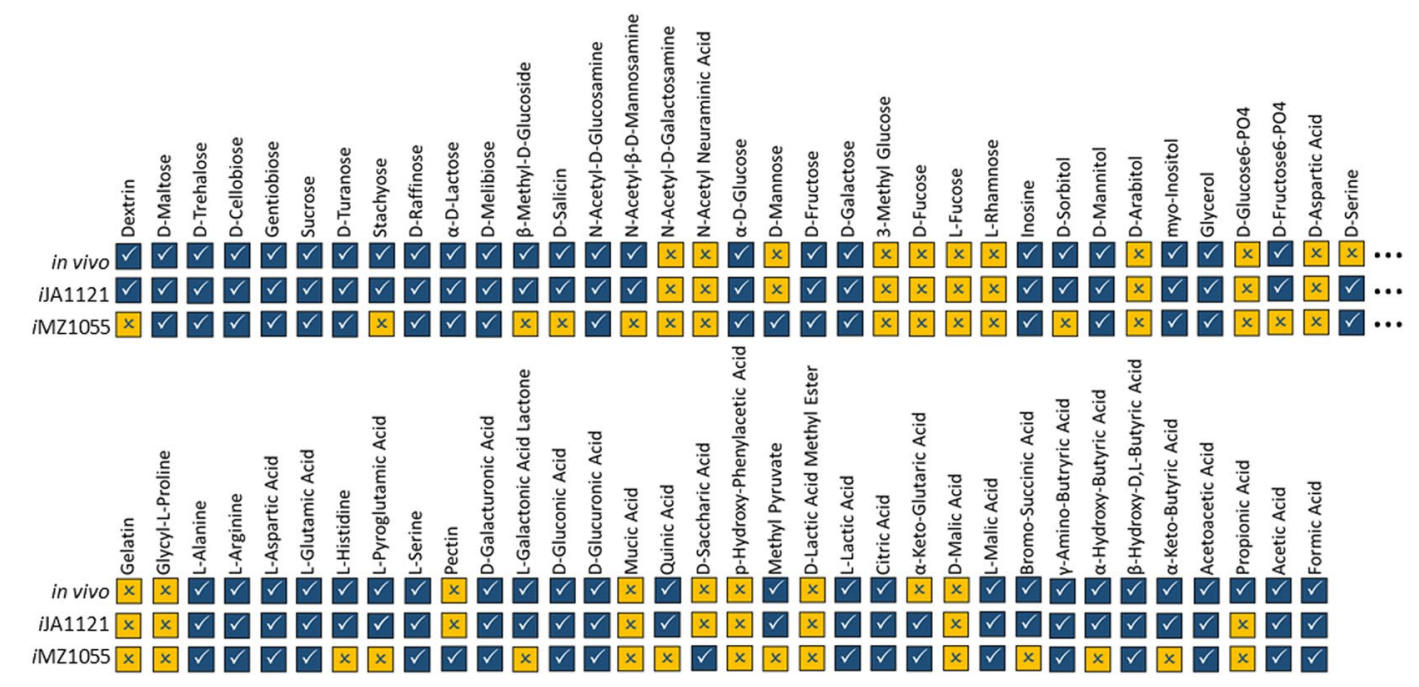

Figure 3. Results of in silico and phenotyping experiments based on growth on 69 different carbon sources ( $\square$ : Growth/True, 凶: No-Growth/False). The experimental results were obtained for Bacillus megaterium DSM319 based on the procedure given in the Materials and Methods.

intracellular metabolites, a literature review was performed seeking any reported gene-protein associations for the missing 9 membrane transporters. The result was used for homology searches in $B$. m. DSM319. Thus, we identified genes encoding uncharacterized membrane proteins responsible for transport of L-pyroglutamic acid (PA) and D-salicin metabolites, and added the relevant transport reactions to the model. For the other 7 intracellular metabolites, interim hypothetical transport reactions were correspondingly added to the model. A hypothetical transport reaction was preserved in the model only when simulating growth on the particular corresponding carbon source. The addition of those hypothetical transport reactions improved model accuracy in all 7 cases. Our results indicated that 56 out of 69 in silico predictions for growth on different carbon sources were compatible with phenotyping assays. Overall, 14 discrepancies were fixed by changing reaction reversibility or filling the gaps based on literature mining (see Table S1 in Supplementary Information). For example, in silico simulations were initially not able to correctly predict the capability of B. m. DSM319 to metabolize PA. In the draft network, PA had been considered as an intracellular metabolite. Our investigations showed that there was a need to add the pyroglutamase (ATP-hydrolyzing) reaction encoded by BMD_2469. Also, it was reported that the uncharacterized membrane proteins DUF969 and DUF979 are involved in PA transport in B. subtilis ${ }^{33,34}$. Based on the protein homology search, we decided to add a transport reaction and assumed that it is encoded by BMD_1100 and BMD_1101. The addition of these two reactions resulted in the model becoming capable of accurately predicting growth on PA as the sole carbon source. By refining and filling in the gaps, the GEM, which will be referred to as iJA1121, reached almost 96\% accuracy in predicting substrate utilization (see Supplementary Information for the model in SBML format). It should be noted that $i$ JA1121 was checked using Memote ${ }^{35}$.

By comparison, out of 69 different carbon sources, $i$ MZ1055, the previous GEM for another strain of $B$. megaterium, predicted growth for only 38 carbon components, where 21 predictions were not consistent with the phenotyping experimental data. Overall, $i$ MZ1055 could estimate growth for about $70 \%$ of carbon sources. Although this outcome could be attributed to metabolic differences between strains and their ability to grow on different carbon sources, it might in part be due to possible deficiencies within iMZ1055. A large number of non-growth/growth (in silico/in vivo) predictions (Fig. 3) demonstrates that some cellular functions were neglected in $i$ MZ1055. It seems that there might exist other parallel metabolic pathways and isozymes that were not taken into account in $i \mathrm{MZ} 1055$.

Constraint-based metabolic model of $B$. $m$. DSM319 and its performance in predicting metabolic behavior. The final GEM of B. m. DSM319, iJA1121, includes 1121 genes, 1349 metabolites, and 1709 reactions. Table 1 presents an overall comparison of GEMs available for Bacillus species. Compared to iMZ1055, i.e., the previous B. megaterium model, iJA1121 includes an increased number of metabolites, reactions, and genes.

We inspected the differences between iJA1121 and iMZ1055, in order to better understand the different metabolic capabilities of the two B. megaterium strains. The results of this study are summarized in Fig. 4. We classified the reactions into 7 categories based on their metabolic subsystems. In all the metabolic subsystems, the number of reactions in $i \mathrm{JA1} 121$ is larger than in $i \mathrm{MZ1055}$. The largest difference is observed for the fatty acid and lipid subsystem, where the number of reactions is approximately six-fold higher in $i$ JA1121. In $i$ MZ1055, teichoic acids and fatty acids were presented as lumped reactions with lumped species. However, $i$ JA1121 explicitly takes into account the reactions producing fatty acids and lipids. In most other metabolic subsystems, the situation was similar. Specifically, iJA1121 includes more reactions for cell wall and capsule synthesis, metabolism of amino acids, carbohydrates, cofactors, and vitamins. This considerable difference in the number of reactions prompted us to perform an in-depth investigation of the type of differences between the two models. All the reactions in iJA1121 were categorized into three classes, based on how they were added to the GEM (Fig. 4b): "No-change" 


\begin{tabular}{|l|l|l|l|l|l|l|}
\hline Model & $\boldsymbol{i J A 1 1 2 1}$ & $\boldsymbol{i M Z 1 0 5 5}$ & $\boldsymbol{i B s u 1 1 4 7}$ & $\boldsymbol{i B s u 1 1 0 3}$ & Oh et al., 2007 & $\boldsymbol{i W X 1 0 0 9}$ \\
\hline Species name & $\begin{array}{l}\text { B. } m . \\
\text { DSM319 }\end{array}$ & $\begin{array}{l}\text { B. } m \text {. } \\
\text { WSH002 }\end{array}$ & B.s. 168 & B.s. 168 & B. s. 168 & B.l. WX02 \\
\hline Reactions & 1709 & 1112 & 1742 & 1443 & 1020 & 1762 \\
\hline Metabolites & 1349 & 993 & 1456 & 1145 & 988 & 1141 \\
\hline Genes & 1121 & 1055 & 1147 & 1103 & 844 & 1009 \\
\hline Transport reactions & 190 & 196 & 290 & 205 & 232 & 176 \\
\hline Refs. & This study & 28 & 58 & 59 & 70 & 60 \\
\hline
\end{tabular}

Table 1. Bacillus species genome-scale metabolic models overview.
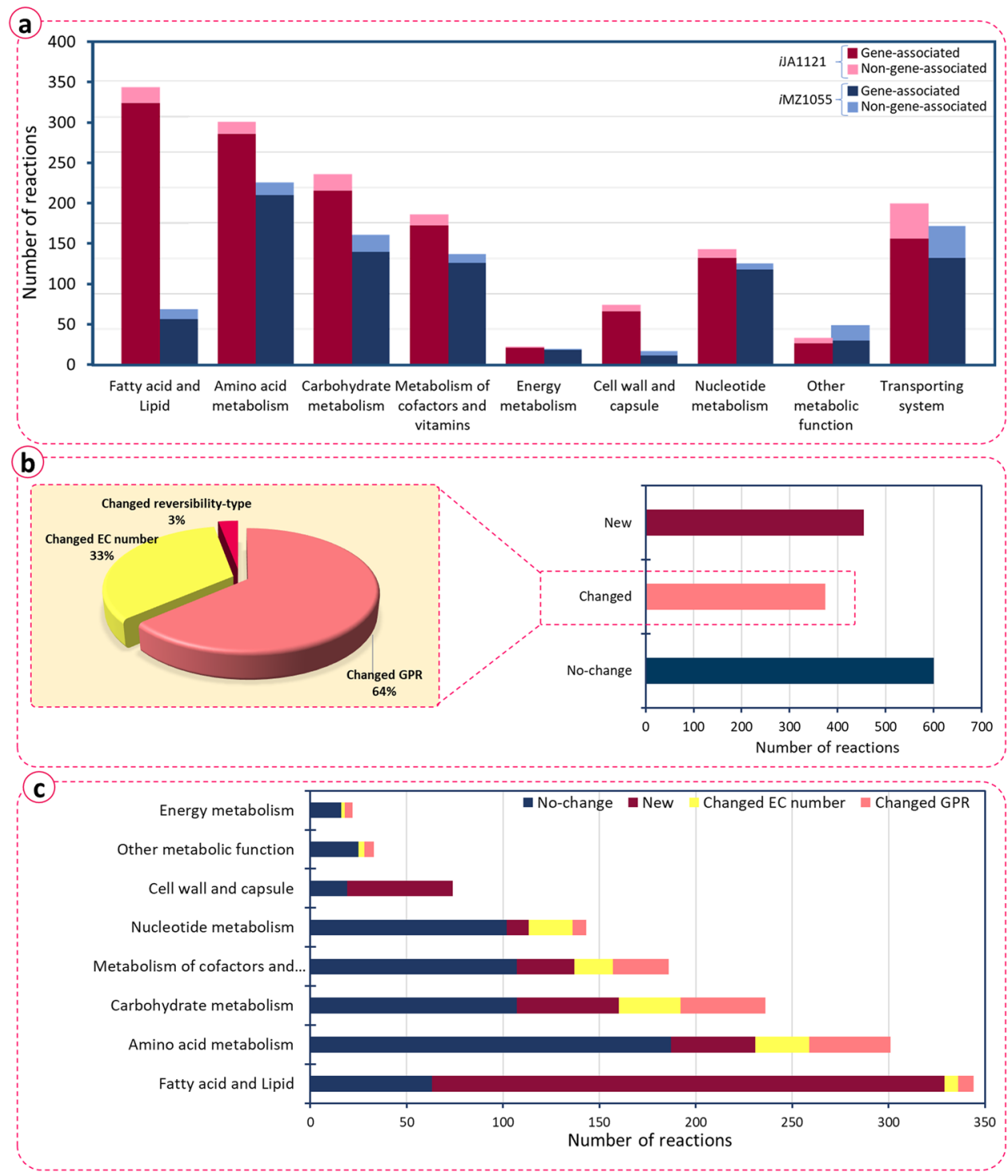

Figure 4. Features of $i$ JA1121, (a) distribution of $i$ JA1121 and $i$ MZ1055 reactions in different subsystems, (b) classification of reactions in $i$ JA1121 based on the modifications, (c) modifications made to the draft network ranked in different subsystems. "No-change" refers to those reactions that were added directly from iMZ1055 based on homology. "Changed" represents those reactions that were added from iMZ1055 based on homology, but with alterations in their GPR associations, EC numbers and/or reversibility-type. "New" refers to those reactions which were newly added during the reconstruction process.

refers to those reactions that remained unchanged, i.e., were added directly from $i \mathrm{MZ1055}$ based on homology. "Changed" represents those reactions added from iMZ1055 based on homology, but with alterations in their GPR associations, EC numbers and/or reversibility-type. Finally, "New" refers to those reactions which were newly added during the reconstruction process. Accordingly, $42 \%$ of the reactions in $i$ JA1121 are added directly 


\begin{tabular}{|l|l|l|l|l|l|l|}
\hline Specific growth rate & Unit & DSM319 & MS941 & WH320 & WH323 \\
\hline & $1 / \mathrm{h}$ & 0.106 & 0.11 & 0.426 & 0.096 & 0.107 \\
\hline Glucose uptake rate & $\mathrm{mmol} / \mathrm{g}_{B M} / \mathrm{h}$ & 1.52 & 1.62 & 5.17 & 1.31 & 1.53 \\
\hline Acetate uptake rate & $\mathrm{mmol} / \mathrm{g}_{B M} / \mathrm{h}$ & 0.15 & 0.17 & 0.6 & 0.17 & 0.16 \\
\hline
\end{tabular}

Table 2. Simulation conditions for different B. $m$. DSM319 strains.

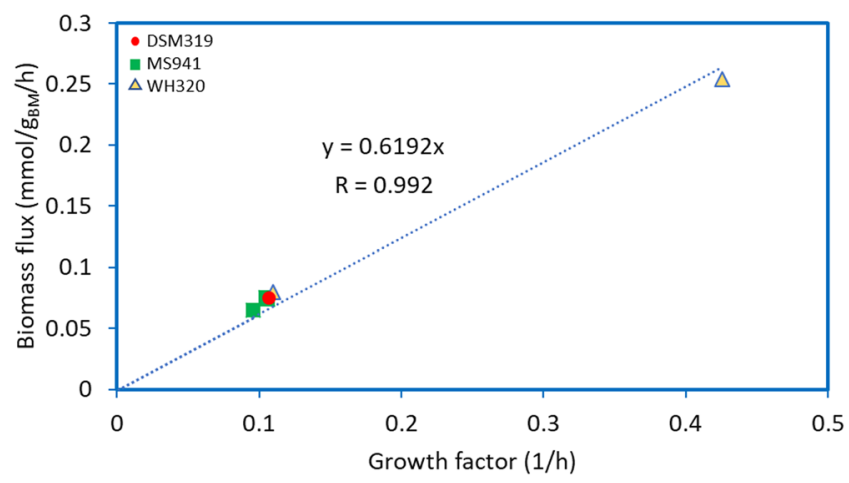

Figure 5. Results of growth simulations under different glucose uptake fluxes. The linear equation intercept is forced to zero (Pearson $\mathrm{R}=0.99, p$-value $=1.2 \mathrm{e}-05$ ).

("No-change"), based on the information in biochemical databases and literature. Among the alterations that were made for the reactions in the "Changed" group that comprised $26 \%$ of the reactions, the changes of GPR associations were the most common. This is presumably due to the automatic annotation of genes by the modelSEED pipeline ${ }^{28}$. In addition, some of the EC numbers of $i$ MZ1055 were obsolete, i.e. they were removed in the most recent release of the KEGG database. The other $32 \%$ of the reactions were those that were not included in $i$ MZ1055. Fig. 4c shows how the changes are distributed over each of the metabolic subsystems. As expected, subsystems with more ambiguity in biochemical databases bore more changes ${ }^{36}$. From Fig. $4 \mathrm{c}$, it can be observed that fatty acid and lipid, as well as cell wall and capsule subsystems have the highest ratio of added reactions. Also, amino acid metabolism, carbohydrate metabolism, metabolism of cofactor and vitamins, and nucleotide metabolism subsystems contained a large number of changes. These changes were based on the up-to-date information in biochemical databases and the literature data, and resulted in addition of new reactions and alterations of several EC numbers and GPR associations. On the other hand, fewest changes in terms of the newly added reactions occurred in the energy metabolism subsystem. In this subsystem, most changes were related to reaction parameters, including some EC numbers and some GPR associations.

Prediction of growth for DSM319 and its derivatives. We characterized the growth behavior of B. $m$.

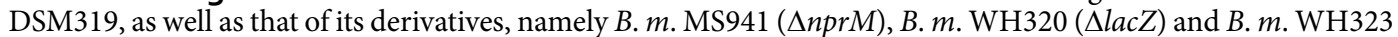
(XylA1-spoVG-lacZ) (Table 2) ${ }^{37}$. All strains were cultivated in M9 medium with glucose as the sole carbon source, under aerobic chemostat conditions. Using FBA, the growth rates were calculated for the mentioned conditions. Mutations in the derivative strains were reported not have a remarkable influence on glucose-based growth ${ }^{37,38}$. To simulate the growth of B. m. DSM319 in the M9 medium, the lower boundary for all exchange reactions, except those related to the metabolites in the M9 medium, was set to zero. There was a significant correlation between the simulation results and the experimental data (Fig. 5); indicating an acceptable agreement between the two (Pearson $\mathrm{R}=0.99, p$-value $=1.2 \times 10^{-05}$ ).

Further investigation of the growth behavior of B. megaterium mutants was carried out by applying the results reported by Wang et al. ${ }^{16}$ to the analysis of MS941 and WH320 strains under high cell density conditions. To estimate glucose uptake fluxes and growth rates, Eqs. (1) and (2) were applied ${ }^{39}$.

$$
\begin{gathered}
v_{g l c, i}=\frac{\mu_{i}\left(C_{g l c, i+1}-C_{g l c, i}\right)}{x_{i}\left(\exp \left(\mu_{i} \times \Delta t_{i}\right)-1\right)} \\
\mu_{i}=\frac{\ln \left(x_{i+1} / x_{i}\right)}{\Delta t_{i}}
\end{gathered}
$$

$v_{g l c}, \mu, \mathrm{C}_{g l c}, x$ and $\Delta t$ are glucose uptake rate, specific growth rate, glucose concentration, biomass concentration and time period, respectively. Subscript $i$ refers to the time step.

We simulated these conditions by running FBA in the minimal medium and allowing glucose to enter the system at the flux obtained by Eq. (1). Simulations were performed under the assumption that both strains have similar growth behavior ${ }^{16}$. There was a good agreement between the biomass flux rates derived from simulations and the growth rate values (Fig. 6) (Pearson $\mathrm{R}=0.994$, $p$-value $=10^{-04}$ ). 


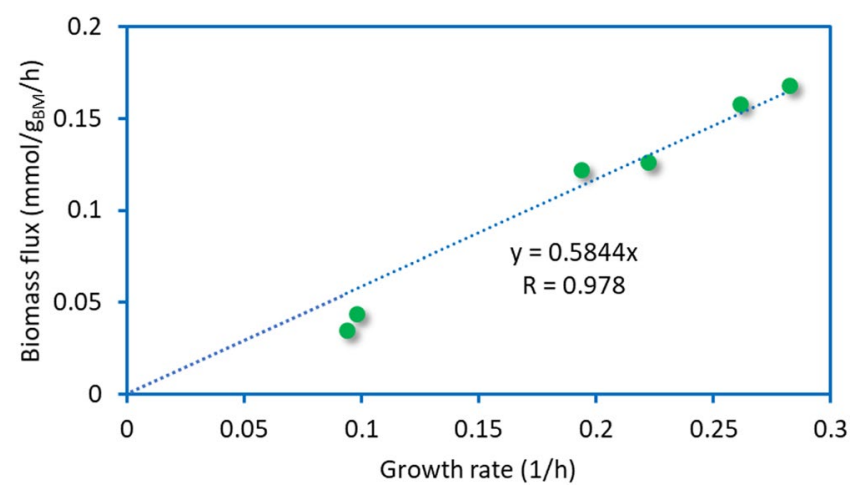

Figure 6. Comparison of biomass production flux obtained by FBA and experimental growth rate values. The linear equation intercept is forced to zero (Pearson $\mathrm{R}=0.994$, $p$-value $=1 \mathrm{e}-4$ ).

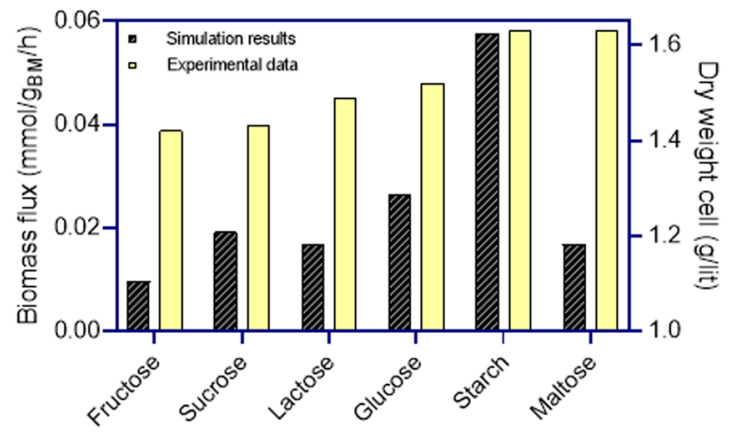

Figure 7. Comparison of biomass rate obtained by FBA and biomass dry cell experiments reported in the literature for different carbon sources including fructose, sucrose, lactose, glucose, starch, and maltose.

Simulation of aroK mutant and shikimic acid production. Limited availability and high price of precursors like shikimic acid and quinic acid is the main restraining factor for industrial production of essential aromatic compounds ${ }^{40}$. As a prominent compound in the pharmaceutical industry, shikimic acid is the main precursor for the synthesis of oseltamiviri ${ }^{14}$. It is reported that a $\Delta$ aroK mutant of B. megaterium can promote shikimate production ${ }^{41}$. The aroK gene encodes shikimate kinase, which catalyzes the bioconversion of shikimate to shikimate-3-phosphate in an ATP-dependent phosphorylation reaction.

We simulated the $\triangle a r o K$ by setting the lower and upper bounds of the associated reaction to zero. Then, we analyzed the effect of different carbon sources on the growth behavior of the mutant strain by setting the lower bounds of the medium components and the carbon source to -1000 and $-5 \mathrm{mmol} / \mathrm{g}_{\mathrm{BM}} / \mathrm{h}$, respectively. There was a significant correlation $(R=0.63, p$-value $<0.05$ in the Pearson correlation test) between biomass flux rate predictions by the GEM and the dry weight cell experiments (Fig. 7). When maltose was used as the sole carbon source, the experimental data and the simulation result were not in agreement. Excluding maltose from the results improved the correlation $(\mathrm{R}=0.946, p$-value $<0.05$ in the Pearson correlation test). This observation suggests that the reactions for assimilation of maltose in the GEM need to be improved.

Further simulations were performed to model the impact of different carbon sources on the growth rate of $\Delta$ aroK strain (Fig. 8). By employing the experimental data reported in the literature ${ }^{41}$ and also applying Eqs. (1) and (2), we ran 43 FBA simulations under the aforementioned conditions. For each simulation, the lower bound of one carbon source was set based on the value obtained by Eq. (1). Then, FBA was performed to find the optimal growth rate. In the following step, we assumed the growth rate to be $\geq 90 \%$ of its maximum value (obtained in the former simulation), and another FBA was performed by taking the flux through shikimate dehydrogenase reaction as the objective function.

As demonstrated in Fig. 8b, this analysis yielded a significant Pearson correlation between predicted growth rate and the experimental cell dry weights. Pearson correlation coefficients for all substrates were higher than 0.85 . Fructose, glucose, and lactose had the highest Pearson correlation coefficients. These findings confirm the ability of $i J A 1121$ to predict mutant growth behavior.

We also investigated the potential of the model for predicting shikimic acid production by the $\Delta a r o K$ mutant. From Fig. 8c, one can observe that in silico shikimic acid production rates are reasonably consistent with the experimental data (for more information see Fig. S1 in Supplementary Information). For starch, maltose, and lactose, the highest Pearson correlation coefficients were observed, ranging from 0.98 to 0.71 , while for glucose the smallest Pearson correlation coefficient, 0.37 , was observed. However, the model failed to predict the shikimic acid production when sucrose was used as the sole carbon source. This can be attributed to different possible reasons. Sucrose has been reported to influence gene expression as a signal molecule in some microorganisms $\mathrm{s}^{42,43}$. 

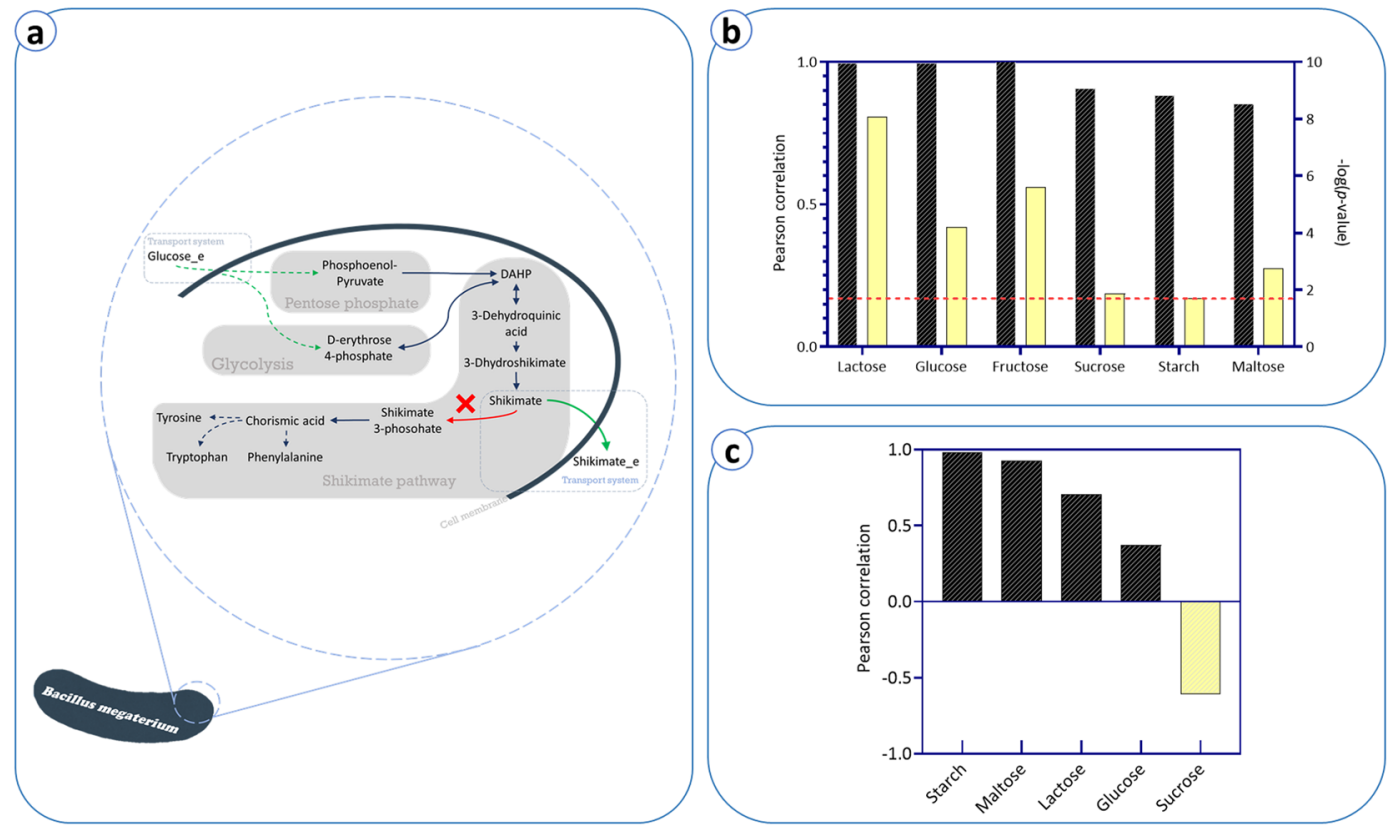

Figure 8. (a) Schematic of the shikimate production pathway. (b) Growth behavior analysis for aroK knock out mutant on different carbon sources. Pearson correlation coefficients and the - $\log (p$-value) represent the results of the comparison study between in silico results and experimental data. The dashed line indicates the minimum $-\log (p$-value $)$ which was obtained for starch uptake. (c) Shikimate production simulations for aroK knock out mutant under different carbon sources.

Also, differences in the metabolism of strains or possible errors in the reported experimental data could be other potential reasons for this discrepancy. These need further investigation.

Formaldehyde inhibitory effect on growth. Formaldehyde $\left(\mathrm{CH}_{2} \mathrm{O}\right)$ is a prominent disinfectant that hinders microbial growth, particularly for Bacillus species, e.g., B. megaterium and B. subtilis ${ }^{44}$. It was shown that the GEMs generated for Bacillus species are incapable of predicting growth behavior under these conditions ${ }^{29}$. Herein, we simulated the glucose/formaldehyde co-metabolism using FBA for growth in minimal medium and glucose as the carbon source. For the formaldehyde uptake simulation, an exchange reaction was added to the model. In order to investigate the effect of formaldehyde addition, its flux was raised in a step-wise manner, and a number of FBAs were performed. The same simulations were run for the previous GEMs for B. megaterium ( $i \mathrm{MZ1055})$ and B. subtilis (iBsu1103) starting from similar dilution rates. Fig. 9 depicts the result of formaldehyde metabolism analysis for Bacillus species. While $i$ MZ1055 and $i$ Bsu 1103 show co-metabolism of glucose/ formaldehyde as these GEMs contain pathways for assimilation of formaldehyde as a carbon source. However, $i J A 1121$ was able to predict formaldehyde sensitivity in B. megaterium. From $i$ JA1121 predictions, by increasing the formaldehyde uptake flux, the biomass production rate remained constant.

Metabolic flux analysis using $\left[\mathrm{U}-{ }^{13} \mathrm{C}\right]$ glucose. Results of FBA simulations typically do not reflect the exact metabolic behavior due to the existence of multiple optimal solutions ${ }^{45}$. In other words, alternate optimal solutions are independent flux distributions that optimize the objective function due to the existence of alternative biochemical pathways ${ }^{46,47}$. Using FVA (Flux Variability Analysis), one can predict the possible range of each flux under a certain optimal growth condition. Therefore, we ran four FVA simulations to investigate how the simulated fluxes agree with experimentally-measured ${ }^{13} \mathrm{C}$ flux data ${ }^{37}$. The results are shown in Fig. 10.

In the simulations, it was assumed that glucose is taken up by B. megaterium directly, as isotopic measurements could not identify which glucose uptake pathway was active ${ }^{47}$. When comparing in silico results with experimental data, suboptimal FVA is more relevant, as it allows the objective function to be within an allowable range (e.g., $\geq 90 \%$ of maximum biomass production rate $)^{48}$.Suboptimal FVA simulations were performed by setting the biomass producing reaction to $90 \%$ of the maximal value obtained by FBA. In Table 3 the reactions are listed. As can be seen in Fig. 10, predictions are in good agreement with experimental data and ${ }^{13} \mathrm{C}$ fluxes generally fall within the intervals suggested by FVA.

There are several possible glucose assimilation mechanisms defined in the model. The predicted interval for the conversion of glucose to glucose-6-phosphate which is limited to $[0,100]$ confirms the existence of alternative reactions for assimilation of glucose. In the predictions, there are some intervals where their maximal or minimal values are found to be unbound. This pertains, for example, to the conversion of glucose-6-phosphate to fructose-6-phosphate, PEP to pyruvate, pyruvate to acetyl-CoA, pyruvate to malate to oxaloacetate. The appearance of these conditions is presumably related to the existence of futile cycles that are often inevitable in reconstructing a GEM. Such futile cycles linked by malate dehydrogenase, pyruvate carboxylase, and the malic enzyme 


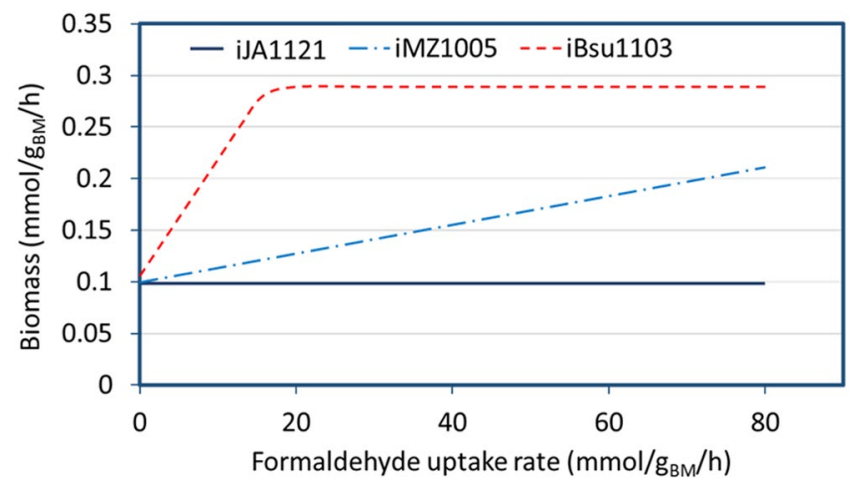

Figure 9. Results of formaldehyde sensitivity simulations in B. megaterium and B. subtilis using iJA1121, $i \mathrm{MZ1055}$, and $i \mathrm{BSU} 1103$. For the simulations, formaldehyde flux was raised in a step-wise manner.

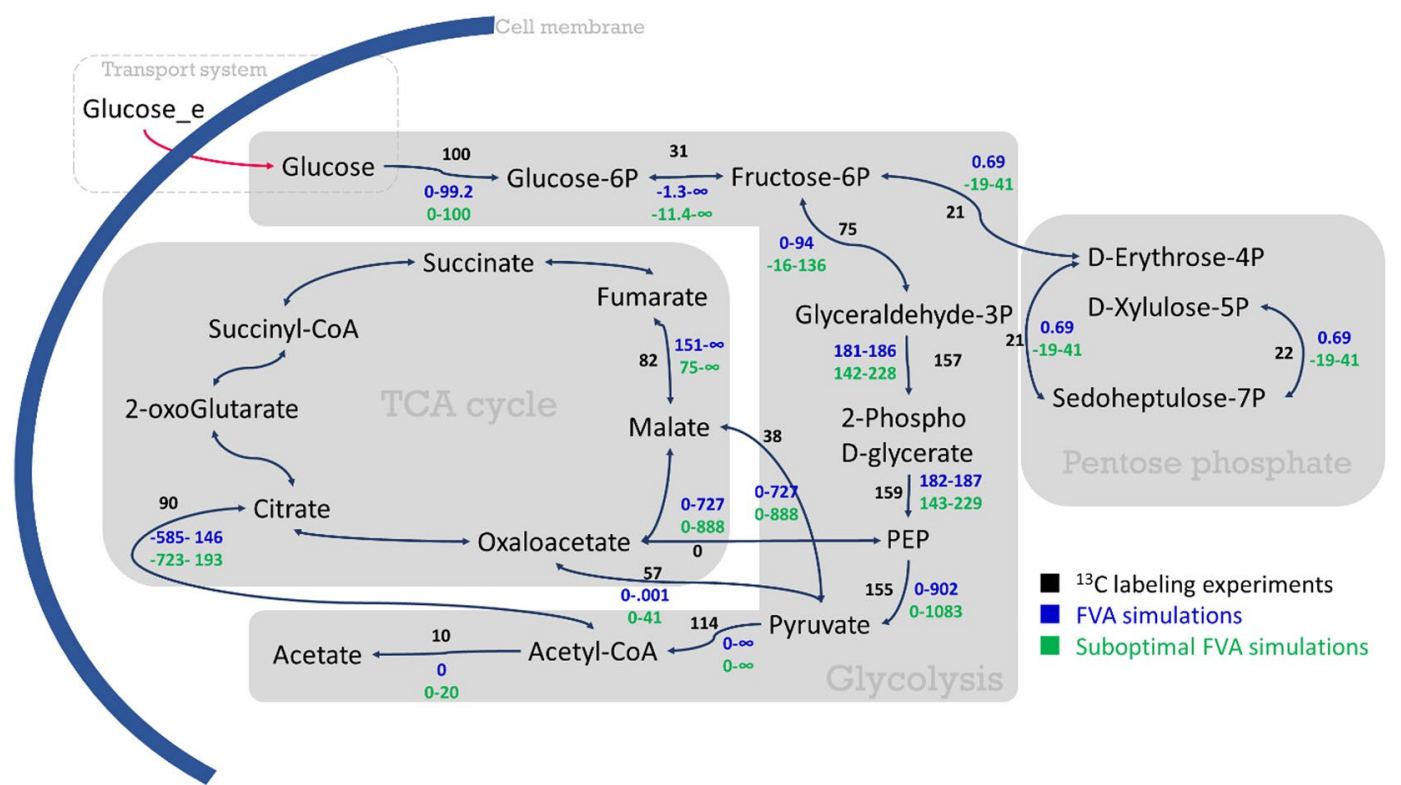

Figure 10. Comparison of FVA and suboptimal FVA simulations as well as ${ }^{13} \mathrm{C}$ labeling experiments. Values in the figure are normalized with respect to the extracellular glucose uptake flux in percent. For a specific reaction, black values are the experimental ${ }^{13} \mathrm{C}$ fluxes, blue values are the flux ranges obtained by FVA simulation and green values are intervals related to the suboptimal FVA simulation.

were investigated experimentally ${ }^{37}$. The occurrence of futile cycles enhances the versatility of the organism to survive in a changing environment. Futile cycles could act as alternative pathways in order to regulate the cellular metabolism of the organism to function optimally ${ }^{49}$. Overall, there is an acceptable agreement between the in silico results and the ${ }^{13} \mathrm{C}$ labeling experiments (for more information see Fig. S2 in Supplementary Information).

Effect of carbon sources on fatty acid biosynthesis and lipid accumulation in B. megaterium. Acetyl-CoA as the precursor of fatty acids plays an important role in the metabolic network (see Fig. 11b). In cellular metabolism, a fraction of pyruvate is converted to acetyl-CoA, which is the main precursor for fatty acids and biosynthesis of lipids ${ }^{50}$. Acetyl-CoA carboxylase catalyzes the conversion of acetyl-CoA to malonyl-CoA. The next metabolic step, transfer of malonyl-CoA to malonyl-[acyl-carrier-protein (ACP)] is catalyzed by [ACP]-S-malonyl transferase, and this initiates the synthesis of fatty acids. Then, malonyl-ACP reacts either with an acetyl-CoA for imitation, or with acyl-CoA primers for elongation, resulting in ketoacyl-ACPs, leading to a process known as the elongation cycle. The elongation cycle includes two NADPH-dependent reduction steps accompanying by a dehydratase reaction ${ }^{51}$.

We investigated the effect of using different carbon sources on fatty acid biosynthesis and lipid accumulation by comparing turnover rates of metabolites in the metabolic network. To do so, a flux-sum analysis was performed ${ }^{52}$. We compared the flux-sum values of metabolites in the network and the results are illustrated in Fig. 11. Four commonly used carbon sources reported in the literature (glucose, glycerol, sucrose, and xylose), were selected to run the simulations. The uptake flux rate of each carbon source was limited to 1 (mmol carbon) $/ \mathrm{g}_{\mathrm{BM}} / \mathrm{h}$ as the basis. Then, the flux-sum of the metabolites was calculated, as explained in the Materials and 


\begin{tabular}{|l|l|l|}
\hline No. & Reaction & $\begin{array}{l}\text { Does the experimental value fall } \\
\text { within the predicted interval? }\end{array}$ \\
\hline 1 & $\mathrm{G} 6 \mathrm{P}[\mathrm{c}] \leftrightarrow \mathrm{F} 6 \mathrm{P}[\mathrm{c}]$ & Yes \\
\hline 2 & $\mathrm{ATP}[\mathrm{c}]+\mathrm{GLC}[\mathrm{c}] \rightarrow \mathrm{H}[\mathrm{c}]+\mathrm{ADP}[\mathrm{c}]+\mathrm{G} 6 \mathrm{P}[\mathrm{c}]$ & Yes \\
\hline 3 & $2 \mathrm{PG}[\mathrm{c}] \leftrightarrow \mathrm{H} 2 \mathrm{O}[\mathrm{c}]+\mathrm{PEP}[\mathrm{c}]$ & Yes \\
\hline 4 & $\mathrm{H}[\mathrm{c}]+\mathrm{ADP}[\mathrm{c}]+\mathrm{PEP}[\mathrm{c}] \rightarrow \mathrm{PYR}[\mathrm{c}]+\mathrm{ATP}[\mathrm{c}]$ & Yes \\
\hline 5 & $\mathrm{E} 4 \mathrm{P}[\mathrm{c}]+\mathrm{XUL} 5 \mathrm{P}[\mathrm{c}] \leftrightarrow \mathrm{T} 3 \mathrm{P} 1[\mathrm{c}]+\mathrm{F} 6 \mathrm{P}[\mathrm{c}]$ & Yes \\
\hline 6 & $\mathrm{~T} 3 \mathrm{P} 1[\mathrm{c}]+\mathrm{S} 7 \mathrm{P}[\mathrm{c}] \leftrightarrow \mathrm{E} 4 \mathrm{P}[\mathrm{c}]+\mathrm{FP}[\mathrm{c}]$ & Yes \\
\hline 7 & $2 \mathrm{PG}[\mathrm{c}] \leftrightarrow 3 \mathrm{PG}[\mathrm{c}]$ & Yes \\
\hline 8 & $\mathrm{FDP}[\mathrm{c}] \leftrightarrow \mathrm{T} 3 \mathrm{P} 2[\mathrm{c}]+\mathrm{T} 3 \mathrm{P} 1[\mathrm{c}]$ & Yes \\
\hline 9 & $\mathrm{XUL} 5 \mathrm{P}[\mathrm{c}]+\mathrm{R} 5 \mathrm{P}[\mathrm{c}] \leftrightarrow \mathrm{T} 3 \mathrm{P} 1[\mathrm{c}]+\mathrm{S} 7 \mathrm{P}[\mathrm{c}]$ & Yes \\
\hline 10 & $2 \mathrm{OFER}[\mathrm{c}]+\mathrm{PYR}[\mathrm{c}]+\mathrm{COA}[\mathrm{c}] \rightarrow 2 \mathrm{RFER}[\mathrm{c}]+\mathrm{ACCOA}[\mathrm{c}]+\mathrm{CO} 2[\mathrm{c}]+2 \mathrm{H}[\mathrm{c}]$ & Yes \\
\hline 11 & $\mathrm{ATP}[\mathrm{c}]+\mathrm{AC}[\mathrm{c}]+\mathrm{COA}[\mathrm{c}] \leftrightarrow \mathrm{ADP}[\mathrm{c}]+\mathrm{ACCOA}[\mathrm{c}]+\mathrm{PI}[\mathrm{c}]$ & Yes \\
\hline 12 & $\mathrm{PYR}[\mathrm{c}]+\mathrm{ATP}[\mathrm{c}]+\mathrm{HCO} 3[\mathrm{c}] \rightarrow \mathrm{H}[\mathrm{c}]+\mathrm{OA}[\mathrm{c}]+\mathrm{ADP}[\mathrm{c}]+\mathrm{PI}[\mathrm{c}]$ & No \\
\hline 13 & $\mathrm{H} 2 \mathrm{O}[\mathrm{c}]+\mathrm{OA}[\mathrm{c}]+\mathrm{ACCOA}[\mathrm{c}] \leftrightarrow \mathrm{H}[\mathrm{c}]+\mathrm{COA}[\mathrm{c}]+\mathrm{CIT}[\mathrm{c}]$ & Yes \\
\hline 14 & $\mathrm{H} 2 \mathrm{O}[\mathrm{c}]+\mathrm{FUM}[\mathrm{c}] \leftrightarrow \mathrm{MAL}[\mathrm{c}]$ & Yes \\
\hline 15 & $\mathrm{NAD}[\mathrm{c}]+\mathrm{MAL}[\mathrm{c}] \rightarrow \mathrm{PYR}[\mathrm{c}]+\mathrm{NADH}[\mathrm{c}]+\mathrm{CO} 2[\mathrm{c}]$ & Yes \\
\hline 16 & $\mathrm{ATP}[\mathrm{c}]+\mathrm{OA}[\mathrm{c}] \rightarrow \mathrm{CO} 2[\mathrm{c}]+\mathrm{ADP}[\mathrm{c}]+\mathrm{PEP}[\mathrm{c}]$ & Yes \\
\hline
\end{tabular}

Table 3. Metabolic reactions used to carry out the FVA simulations.

Methods section. Fig. 11a illustrates the flux-sum results for the metabolites, normalized with the maximum value of each metabolite. Although most of the metabolites show similar levels for different carbon sources, glycerol has a higher flux-sum distribution and xylose represents the lowest flux-sum values for most of the compounds. The higher the utilization of central metabolism, the higher the production of the biomass precursors. Glycerol with high flux-sum values for amino acids and glycolytic intermediates could potentially be a favorite source for fermentation products such as poly-hydroxybutyrate ${ }^{53}$ and fatty acids ${ }^{54,55}$. High turnover rates of amino acids known to be the sources of acyl-CoAs (leucine, isoleucine, and valine) indicate active biosynthesis of fatty acids ${ }^{56}$. Besides, growth on glycerol exhibited the highest flux-sum value for acetyl-CoA, which in turn favors lipid accumulation. These findings prompted us to compare the flux-sum values of lipids (Fig. 11c,d). The maximum flux-sum values of lipids and fatty acid intermediates (specified with red boxes in the figures) were applied as the references for normalization. As shown in Fig. 11c, the flux-sum distribution indicates that lipids have comparable flux-sums. It also shows that using glycerol as the carbon source leads to the highest flux-sum values for lipids. In addition, flux-sum values of fatty acids when glycerol is set as the carbon source are maximal (Fig. 11d). Among different types of fatty acids, C15-fatty acid had the highest, while C14- and C17-fatty acids had the lowest flux-sum values. These findings are consistent with the results reported by Scandella and Kornberg ${ }^{57}$ obtained during log-phase growth.

\section{Materials and Methods}

Genome files and genome-scale metabolic models. In order to identify the homologous gene pairs, GenBank genome (.gbk) files for B. m. DSM319, B. $m$ WSH002, B. subtilis 168 (B. s. 168) and B. licheniformis WX02 (B. l. WX02) containing genomic sequences and annotations were downloaded (with accession numbers CP001982.1, CP003017.1, CP010052.1, and AHIF00000000.1, respectively). Four GEMs, $i$ MZ1055 for B. $m$ WSH002 $2^{28}, i$ Bsu $1147^{58}$ and $i$ Bsu $1103^{59}$ for B. subtilis 168 and $i$ WX1009 for B. licheniformis. WX02 ${ }^{60}$ were obtained for comparison and/or were used as the reconstruction templates.

Genome-scale metabolic network reconstruction. As the first step, a homology search was done based on progressive multiple alignments using Mauve version 2.4.0, using its default parameter settings. Mauve is a computational framework for multiple genome alignments in the presence of large-scale evolutionary events ${ }^{30}$. Accordingly, the related GenBank genome files containing genomic sequences and annotations were downloaded to identify gene orthologous pairs using the 'progressiveMauve' tool. Then, based on the results gained by Mauve, the associated reactions in $i \mathrm{MZ1} 1055$ were identified and used as the basis of the draft network. In the second step, the refinement of the draft network was automatically performed (and then manually curated) based on public biochemical databases such as $\mathrm{KEGG}^{61}$, MetaCyc ${ }^{62}$, UniProt ${ }^{63}$, and PATRIC ${ }^{64}$. In the next step, with a similar strategy, we found all of the relevant genes and reactions which were present in $i$ Bsu $1103^{59}, i$ Bsu $1147^{58}$ and $i \mathrm{WX} 1009^{60}$ and their corresponding GenBank genome files. Then, according to the information on TCDB ${ }^{65}$, the transport equations were added to the draft network. Finally, the gaps of the model were identified and corrected, based on the phenotyping results as well as the experimental data reported in the literature.

Flux balance analysis and biomass objective function. FBA is one of the popular methods for analyzing the flux of reactions in metabolic networks under steady-state conditions ${ }^{66,67}$. FBA assumes that no accumulation of metabolites occurs during growth $(d c / d t=0)$, while a cellular objective, typically biomass production rate, is optimized. Based on the FBA, a linear programming problem is defined as follows: 


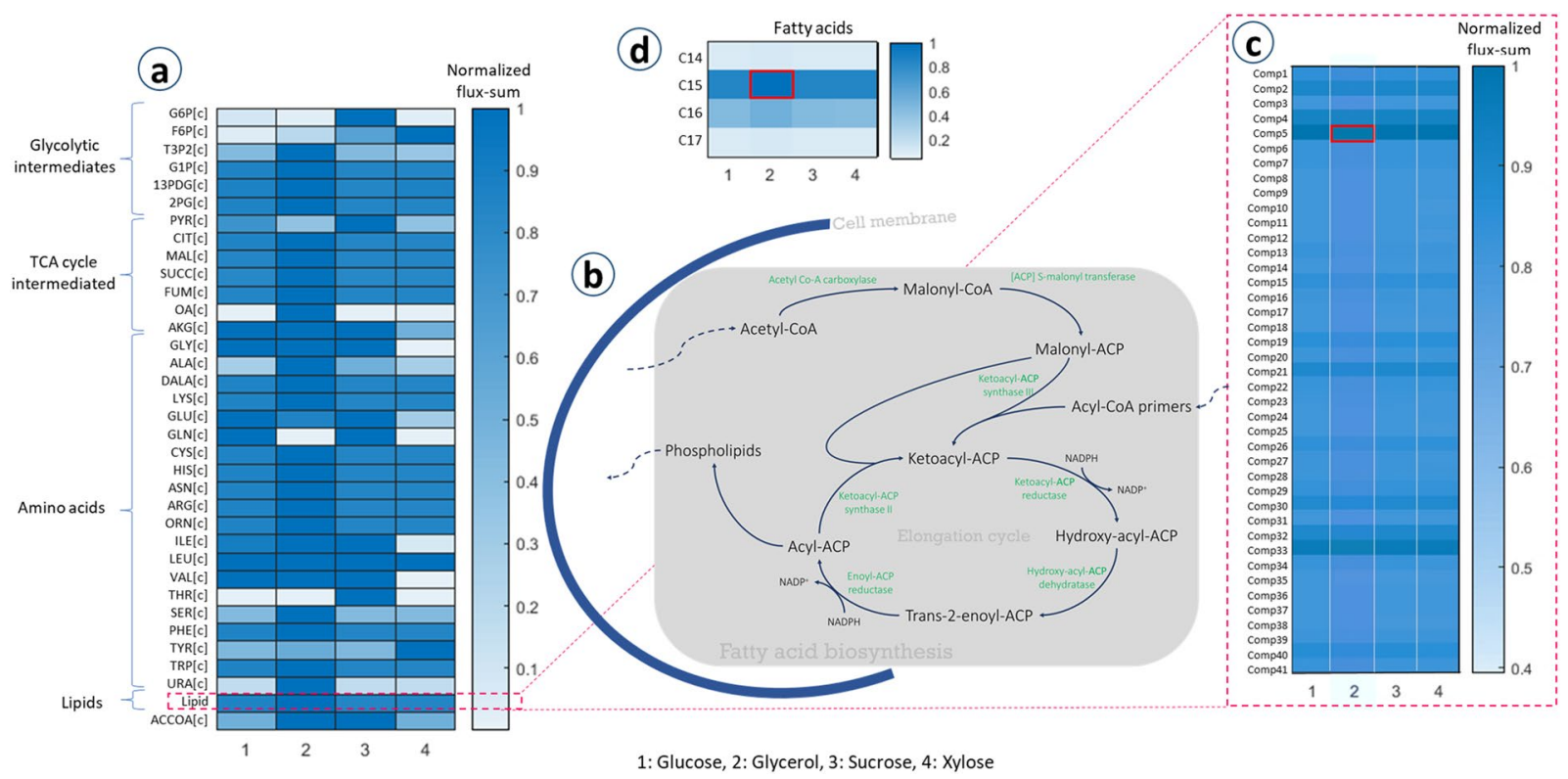

Figure 11. The heatmap representing the results of the flux-sum values of different components including glycolytic intermediates, TCA cycle intermediates, amino acids, and lipids. The comparisons were conducted for different carbon sources. (b) Schematic of fatty acid biosynthesis pathway. (c) Results of flux-sum values of the lipids constituting biomass. (d) Results of flux-sum values of the lipids constituting biomass.

$$
\begin{gathered}
\operatorname{maximize}: Z=\sum_{j} c_{j} v_{j} \\
\text { subject to: } \sum_{j} S_{i j} v_{j}=0 \\
v_{j}^{\max } \leq v_{j} \leq v_{j}^{\max }
\end{gathered}
$$

where $Z$ is a linear function related to the cellular objective and the coefficients $c_{j}$ determine the weights of the reaction $j$. Also, $v_{j}$ refers to the flux rate of the reaction $j$ in the network and $S_{i j}$ is the stoichiometric coefficient of metabolite $i$ in reaction $j$. In this approach, each flux, $v_{i}$ is constrained to the given minimum and maximum values. To perform FBA, a biomass producing reaction, representing a weighted ratio of components in cell dry weight, is maximized based on the evolutionary assumption ${ }^{68,69}$. In this study, the biomass producing reaction of the $i$ MZ1055 model is used, which in turn is based on the biomass composition of the B. subtilis model ${ }^{70}$. Unless stated otherwise, the biomass production rate was used as the objective function in FBA simulations.

Flux variability analysis. FVA is a mathematical tool for finding the minimum and maximum possible fluxes of each reaction, while other constraints are satisfied and the objective function takes the optimal (or, suboptimal) value ${ }^{71}$. In this study, suboptimal FVA was used to find the minimum and maximum possible fluxes of each reaction while the objective function was bound to $90 \%$ of its maximal value (achieved by FBA).

Flux-sum analysis. In order to illuminate the role of metabolite in the network and study turnover rates of the metabolites, we used flux-sum analysis ${ }^{72}$. The flux-sum of metabolite $i$ is defined as the summation of all consumption or generation fluxes as follows ${ }^{52}$ :

$$
\varphi_{i}=\frac{1}{2} \sum_{j}\left|S_{i j} v_{j}\right|
$$

The existence of possible alternate optimal solutions can result in ambiguity in the interpretation of FBA solutions. Thus, to identify the most plausible flux distribution, the minimization of the sum of total fluxes makes the calculation $\varphi_{i}$ feasible ${ }^{73}$.

Experimental methods. To discern the phenotypic pattern of B. m. DSM319, the ability of the strain to grow on different carbon sources was tested using the Biolog GEN III microplate. This was used to analyze the strain under 94 phenotyping tests. As specified by the manufacturer, a pure culture of the strain was incubated at $30^{\circ} \mathrm{C}$ overnight on an agar plate with $5 \%$ sheep blood, and then suspended in a special inoculating fluid at the predetermined cell density (90-98\% Transmittance). Then, $100 \mathrm{ml}$ of the cell suspension was inoculated 
into each well of the GEN III MicroPlate ${ }^{\mathrm{TM}}$. The microplate was incubated at $30^{\circ} \mathrm{C}$ for 24 hours. Readouts of growth-dependent color change were obtained using a microplate reader and interpreted based on the Biolog protocols.

\section{Conclusion}

Aerobic Gram-positive Bacillus species are commonly used in biotechnology, especially in food, pharmaceutical, and environmental processes. Lack of knowledge about growth behavior of these organisms complicates the design and monitoring of industrial microbial processes. Generating constraint-based models that can predict growth behavior is an important step in addressing this knowledge gap. In this regard, several GEMs for Bacillus species have been constructed. After the reconstruction of the GEM for B. subtilis 168, generated by Oh et al., in silico models were developed for B. $m$. WSH002 (iMZ1055) and B. l. WX02 (iWX1009). In addition, two other GEMs for B. subtilis 168, $i$ Bsu1103 and $i$ Bsu1147, were published more recently. It has been reported that existing genome-scale models for B. megaterium could not correctly predict the utilization of amino acids and some carbon sources ${ }^{29}$. Herein, we present a GEM for B. $m$. DSM319, iJA1121. The model includes 1709 reactions, 1349 metabolites with 1121 genes. Barring exchange reactions, $91 \%$ of the reactions are gene-associated.

Following an automated approach, the draft network was curated manually by updating the GPR associations and EC numbers as well as adding several reactions. The genome-scale model was validated using our own experimental data on Biolog phenotyping and published data on B. $m$. DSM319 growth on different carbon sources (available on PubMed). FBA and FVA were applied to perform the simulations. Our findings suggested a better agreement of in silico predictions and experimental data for $i$ JA1121. Results of carbon source utilization experiments and the model predictions matched in $96 \%$ cases. Moreover, the growth behavior of different mutant strains of $B . m$. DSM319 was studied and results indicated a very good match between $i J A 1121$ predictions and in vivo data, with very few exceptions. For instance, the simulation of shikimate production in aroK knock out worked well on all tested carbon sources except sucrose. Further investigations were performed by comparing in silico results with ${ }^{13} \mathrm{C}$ labeling experiments. Regarding suboptimal FVA results, the experimental ${ }^{13} \mathrm{C}$ fluxes fell within the predicted intervals. In conclusion, iJA1121 seems to offer a clear improvement over $i$ MZ1055, where the success rate of the previous model in accurately predicting growth behavior in the same type of simulations was only $70 \%$.

Received: 4 June 2019; Accepted: 21 November 2019;

Published online: 10 December 2019

\section{References}

1. Elmerich, C. \& Aubert, J. P. Synthesis of glutamate by a glutamine: 2-oxo-glutarate amidotransferase (NADP oxidoreductase) in Bacillus megaterium. Biochem. Biophys. Res. Commun. 42, 371-376 (1971).

2. Hitchins, A. D., Kahn, A. J. \& Slepecky, R. A. Interference contrast and phase contrast microscopy of sporulation and germination of Bacillus megaterium. J. Bacteriol. 96, 1811-1817 (1968).

3. Santos, S., Neto, I. F. F., Machado, M. D., Soares, H. M. V. M. \& Soares, E. V. Siderophore production by Bacillus megaterium: effect of growth phase and cultural conditions. Appl. Biochem. Biotechnol. 172, 549-560 (2014).

4. Barak, I. et al. Structure and function of the Bacillus SpoIIE protein and its localization to sites of sporulation septum assembly. Mol. Microbiol. 19, 1047-1060 (1996).

5. Vary, P. S. et al. Bacillus megaterium-from simple soil bacterium to industrial protein production host. Applied Microbiology and Biotechnology 76, 957-967 (2007).

6. Martín, L., Prieto, M. A., Cortés, E. \& García, J. L. Cloning and sequencing of the pac gene encoding the penicillin G acylase of Bacillus megaterium ATCC 14945. FEMS Microbiol. Lett. 125, 287-292 (1995).

7. Malten, M., Hollmann, R., Deckwer, W. D. \& Jahn, D. Production and secretion of recombinant Leuconostoc mesenteroides dextransucrase DsrS in Bacillus megaterium. Biotechnol. Bioeng. 89, 206-218 (2005).

8. Lammers, M., Nahrstedt, H. \& Meinhardt, F. The Bacillus megaterium comE locus encodes a functional DNA uptake protein. J. Basic Microbiol. 44, 451-458 (2004).

9. Biedendieck, R. et al. Systems biology of recombinant protein production using Bacillus megaterium. In Methods in Enzymology 500, 165-195 (Elsevier Inc., 2011).

10. Grage, K., McDermott, P. \& Rehm, B. H. A. Engineering Bacillus megaterium for production of functional intracellular materials. Microb. Cell Fact. 16, 211 (2017).

11. Guo, J., Erskine, P., Coker, A. R., Wood, S. P. \& Cooper, J. B. Structural studies of domain movement in active-site mutants of porphobilinogen deaminase from Bacillus megaterium. Acta Crystallogr. Sect. FStructural Biol. Commun. 73, 612-620 (2017).

12. Nahrstedt, H., Wittchen, K.-D., Rachman, M. A. \& Meinhardt, F. Identification and functional characterization of a type I signal peptidase gene of Bacillus megaterium DSM319. Appl. Microbiol. Biotechnol. 64, 243-249 (2004).

13. Ghosh, S. \& Banerjee, U. C. Generation of aroE overexpression mutant of Bacillus megaterium for the production of shikimic acid. Microb. Cell Fact. 14, 69 (2015).

14. Ghosh, S., Pawar, H., Pai, O. \& Banerjee, U. C. Microbial transformation of quinic acid to shikimic acid by Bacillus megaterium. Bioresour. Bioprocess. 1, 7 (2014).

15. Arshadi, M., Mousavi, S. M. \& Rasoulnia, P. Enhancement of simultaneous gold and copper recovery from discarded mobile phone PCBs using Bacillus megaterium: RSM based optimization of effective factors and evaluation of their interactions. Waste Manag. 57, 158-167 (2016).

16. Wang, W., Hollmann, R. \& Deckwer, W.-D. Comparative proteomic analysis of high cell density cultivations with two recombinant Bacillus megaterium strains for the production of a heterologous dextransucrase. Proteome Sci. 4, 19 (2006).

17. Jordan, E. et al. Production of recombinant antibody fragments in Bacillus megaterium. Microb. Cell Fact. 6, 2 (2007).

18. Wang, W. et al. Proteome analysis of a recombinant Bacillus megaterium strain during heterologous production of a glucosyltransferase. Proteome Sci. 3, 4 (2005).

19. Moore, S. J., Mayer, M. J., Biedendieck, R., Deery, E. \& Warren, M. J. Towards a cell factory for vitamin B12 production in Bacillus megaterium: bypassing of the cobalamin riboswitch control elements. N. Biotechnol. 31, 553-561 (2014).

20. Abdulmughni, A. et al. Biochemical and structural characterization of CYP109A2, a vitamin D3 25-hydroxylase from Bacillus megaterium. FEBS J. 284, 3881-3894 (2017).

21. Bäumchen, C. et al. D-Mannitol production by resting state whole cell biotransformation of D-fructose by heterologous mannitol and formate dehydrogenase gene expression in Bacillus megaterium. Biotechnol. J. 2, 1408-1416 (2007). 
22. Hollmann, R. \& Deckwer, W.-D. Pyruvate formation and suppression in recombinant Bacillus megaterium cultivation. J. Biotechnol. 111, 89-96 (2004).

23. Eppinger, M. et al. Genome Sequences of the Biotechnologically Important Bacillus megaterium Strains QM B1551 and DSM319. J. Bacteriol. 193, 4199-4213 (2011).

24. Bordbar, A., Monk, J. M., King, Z. A. \& Palsson, B. O. Constraint-based models predict metabolic and associated cellular functions. Nat. Rev. Genet. 15, 107-120 (2014).

25. Babaei, P., Marashi, S.-A. \& Asad, S. Genome-scale reconstruction of the metabolic network in Pseudomonas stutzeri A1501. Mol. Biosyst. 11, 3022-3032 (2015).

26. Park, J. M., Kim, T. Y. \& Lee, S. Y. Prediction of metabolic fluxes by incorporating genomic context and flux-converging pattern analyses. Proc. Natl. Acad. Sci. USA 107, 14931-14936 (2010).

27. Patil, K. R., Åkesson, M. \& Nielsen, J. Use of genome-scale microbial models for metabolic engineering. Curr. Opin. Biotechnol. 15, 64-69 (2004)

28. Zou, W., Zhou, M., Liu, L. \& Chen, J. Reconstruction and analysis of the industrial strain Bacillus megaterium WSH002 genomescale in silico metabolic model. J. Biotechnol. 164, 503-509 (2013).

29. Ghasemi-Kahrizsangi, T., Marashi, S.-A. \& Hosseini, Z. Genome-scale metabolic network models of Bacillus species suggest that model improvement is necessary for biotechnological applications. Iran. J. Biotechnol. 16, 164-172 (2018).

30. Darling, A. E., Mau, B. \& Perna, N. T. progressiveMauve: multiple genome alignment with gene gain, loss and rearrangement. PLoS One 5, e11147 (2010).

31. Kawashima, S., Katayama, T., Sato, Y., Kanehisa, M. \& KEGG, A. P. I. a web service using SOAP/WSDL to access the KEGG system. Genome Informatics 14, 673-674 (2003).

32. Rau, M. H. \& Zeidan, A. A. Constraint-based modeling in microbial food biotechnology. Biochem. Soc. Trans. 46, 249-260 (2018).

33. Van Der Werf, P. \& Meister, A. The metabolic formation and utilization of 5-oxo-l-proline (L-pyroglutamate, L-pyrrolidone carboxylate). in Advances in Enzymology and Related Areas of Molecular Biology 43, 519-556 (John Wiley \& Sons, Ltd, 1975).

34. Niehaus, T. D., Elbadawi-Sidhu, M., De Crécy-Lagard, V., Fiehn, O. \& Hanson, A. D. Discovery of a widespread prokaryotic 5-oxoprolinase that was hiding in plain sight. J. Biol. Chem. 292, 16360-16367 (2017).

35. Lieven, C. et al. Memote: A community driven effort towards a standardized genome-scale metabolic model test suite. BioRxiv, https://doi.org/10.1101/350991 (2018).

36. Oberhardt, M. A., Puchałka, J., Martins dos Santos, V. A. P. \& Papin, J. A. Reconciliation of genome-scale metabolic reconstructions for comparative systems analysis. PLoS Comput. Biol. 7, e1001116 (2011).

37. Fürch, T., Hollmann, R., Wittmann, C., Wang, W. \& Deckwer, W. D. Comparative study on central metabolic fluxes of Bacillus megaterium strains in continuous culture using 13C labelled substrates. Bioprocess Biosyst. Eng. 30, 47-59 (2007).

38. Fürch, T. et al. Effect of different carbon sources on central metabolic fluxes and the recombinant production of a hydrolase from Thermobifida fusca in Bacillus megaterium. J. Biotechnol. 132, 385-394 (2007).

39. Mahadevan, R., Edwards, J. S. \& Doyle, F. J. Dynamic flux balance analysis of diauxic growth. Escherichia coli, Biophys. J. 83, 1331 (2002).

40. Farina, V. \& Brown, J. D. Tamiflu: the supply problem. Angew. Chemie Int. Ed. 45, 7330-7334 (2006).

41. Ghosh, S., Mohan, U. \& Banerjee, U. C. Studies on the production of shikimic acid using the aroK knockout strain of Bacillus megaterium. World J. Microbiol. Biotechnol. 32, 127 (2016).

42. Nishikawa, F. et al. Effect of sucrose on ascorbate level and expression of genes involved in the ascorbate biosynthesis and recycling pathway in harvested broccoli florets. J. Exp. Bot. 56, 65-72 (2005).

43. Huang, H. et al. Sucrose and ABA regulate starch biosynthesis in maize through a novel transcription factor, ZmEREB156. Sci. Rep. 6, $27590(2016)$

44. Trujillo, R. \& Lindell, K. F. New formaldehyde base disinfectants. Appl. Microbiol. 26, 106-110 (1973).

45. O’Brien, E. J., Monk, J. M. \& Palsson, B. O. Using genome-scale models to predict biological capabilities. Cell 161, 971-987 (2015).

46. Pereira, R., Nielsen, J. \& Rocha, I. Improving the flux distributions simulated with genome-scale metabolic models of Saccharomyces cerevisiae. Metab. Eng. Commun. 3, 153-163 (2016).

47. Puchałka, J. et al. Genome-scale reconstruction and analysis of the Pseudomonas putida KT2440 metabolic network facilitates applications in biotechnology. PLoS Comput. Biol. 4, e1000210 (2008).

48. Chen, T., Xie, Z. \& Ouyang, Q. Expanded flux variability analysis on metabolic network of Escherichia coli. Sci. Bull. 54, 2610-2619 (2009).

49. Diesterhaft, M. D. \& Freese, E. Role of pyruvate phosphoenolpyruvate and malic enzyme during growth and sporulation of Bacillus subtilis. J. Biol. Chem. 245, 6062-6070 (1973).

50. White, S. W., Zheng, J., Zhang, Y.-M. \& Rock, C. O. The structural biology of type II fatty acid biosynthesis. Annu. Rev. Biochem. 74, 791-831 (2005).

51. Diomandé, S. E., Nguyen-The, C., Guinebretière, M.-H., Broussolle, V. \& Brillard, J. Role of fatty acids in Bacillus environmental adaptation. Front. Microbiol. 6, 813 (2015).

52. Kim, P.-J. et al. Metabolite essentiality elucidates robustness of Escherichia coli metabolism. Proc. Natl. Acad. Sci. 104, 13638-13642 (2007).

53. Naranjo, J. M., Posada, J. A., Higuita, J. C. \& Cardona, C. A. Valorization of glycerol through the production of biopolymers: The PHB case using Bacillus megaterium. Bioresour. Technol. 133, 38-44 (2013).

54. Hou, C. T. Effect of environmental factors on the production of oxygenated unsaturated fatty acids from linoleic acids by Bacillus megaterium ALA2. Appl. Microbiol. Biotechnol. 69, 463-468 (2005).

55. Hilker, B. L., Fukushige, H., Hou, C. \& Hildebrand, D. Comparison of Bacillus monooxygenase genes for unique fatty acid production. Prog. Lipid Res. 47, 1-14 (2008).

56. Kaneda, T. Iso- and anteiso-fatty acids in bacteria: biosynthesis, function, and taxonomic significancet. Microbiol. Rev. 55, 288-302 (1991).

57. Scandella, C. J. \& Kornberg, A. Biochemical studies of bacterial sporulation and germination. J. Bacteriol. 98, 8286 (1969).

58. Hao, T. et al. In silico metabolic engineering of Bacillus subtilis for improved production of riboflavin, Egl-237, (R,R)-2,3-butanediol and isobutanol. Mol. Biosyst. 9, 2034-2044 (2013).

59. Henry, C. S., Zinner, J. F., Cohoon, M. P. \& Stevens, R. L. iBsu1103: A new genome-scale metabolic model of Bacillus subtilis based on SEED annotations. Genome Biol. 10, 1-15 (2009).

60. Guo, J., Zhang, H., Wang, C., Chang, J. W. \& Chen, L. L. Construction and analysis of a genome-scale metabolic network for Bacillus licheniformis WX-02. Res. Microbiol. 167, 282-289 (2016).

61. Kanehisa, M. \& Goto, S. KEGG: Kyoto Encyclopedia of Genes and Genomes. Nucleic Acids Res. 28, 27-30 (2000).

62. Karp, P. D., Riley, M., Paley, S. M. \& Pellegrini-Toole, A. The MetaCyc database. Nucleic Acids Res. 30, 59-61 (2002).

63. Bateman, A. et al. UniProt: the universal protein knowledgebase. Nucleic Acids Res. 45, D158-D169 (2017).

64. Wattam, A. R. et al. Improvements to PATRIC, the all-bacterial bioinformatics database and analysis resource center. Nucleic Acids Res. 45, D535-D542 (2017).

65. Saier, M. H., Tran, C. V. \& Barabote, R. D. TCDB: the Transporter Classification Database for membrane transport protein analyses and information. Nucleic Acids Res. 34, D181-D186 (2006). 
66. Tack, I. L. M. M., Nimmegeers, P., Akkermans, S., Hashem, I. \& Van Impe, J. F. M. Simulation of Escherichia coli Dynamics in biofilms and submerged colonies with an individual-based model including metabolic network information. Front. Microbiol. 8, 2509 (2017).

67. Mishra, P. et al. Genome-scale model-driven strain design for dicarboxylic acid production in Yarrowia lipolytica. BMC Syst. Biol. 12, 12 (2018).

68. Oberhardt, M. A., Puchałka, J., Fryer, K. E., Martins Dos Santos, V. A. P. \& Papin, J. A. Genome-scale metabolic network analysis of the opportunistic pathogen Pseudomonas aeruginosa PAO1. J. Bacteriol. 190, 2790-2803 (2008).

69. Feist, A. M. \& Palsson, B. O. The biomass objective function. Curr. Opin. Microbiol. 13, 344-349 (2010).

70. Oh, Y. K., Palsson, B. O., Park, S. M., Schilling, C. H. \& Mahadevan, R. Genome-scale reconstruction of metabolic network in Bacillus subtilis based on high-throughput phenotyping and gene essentiality data. J. Biol. Chem. 282, 28791-28799 (2007).

71. Mahadevan, R. \& Schilling, C. H. The effects of alternate optimal solutions in constraint-based genome-scale metabolic models. Metab. Eng. 5, 264-276 (2003).

72. Chung, B. K. S. \& Lee, D.-Y. Flux-sum analysis: a metabolite-centric approach for understanding the metabolic network. BMC Syst. Biol. 3, 117 (2009).

73. Mishra, P. et al. Genome-scale metabolic modeling and in silico analysis of lipid accumulating yeast Candida tropicalis for dicarboxylic acid production. Biotechnol. Bioeng. 113, 1993-2004 (2016).

\section{Acknowledgements}

This study was financially supported by Tarbiat Modares University under grant number IG-39701. Authors also would like to thank the Biotechnology Development Council of the Islamic Republic of Iran for their support under grant number 970301 . We are grateful to Dr. Kirsten Leistner for language-editing the manuscript.

\section{Author contributions}

Conceived and designed the experiments: J.A. and S.A.M.; Performed the experiments: J.A.; Analyzed the data: J.A. and S.A.M.; Prepared and edited the manuscript: J.A., S.M.M., S.A.M., I.M. and A.J.; Prepared software and resources: I.M. All authors read and approved the manuscript.

\section{Competing interests}

The authors declare no competing interests.

\section{Additional information}

Supplementary information is available for this paper at https://doi.org/10.1038/s41598-019-55041-w.

Correspondence and requests for materials should be addressed to S.M.M. or S.-A.M.

Reprints and permissions information is available at www.nature.com/reprints.

Publisher's note Springer Nature remains neutral with regard to jurisdictional claims in published maps and institutional affiliations.

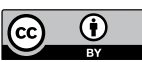

Open Access This article is licensed under a Creative Commons Attribution 4.0 International License, which permits use, sharing, adaptation, distribution and reproduction in any medium or format, as long as you give appropriate credit to the original author(s) and the source, provide a link to the Creative Commons license, and indicate if changes were made. The images or other third party material in this article are included in the article's Creative Commons license, unless indicated otherwise in a credit line to the material. If material is not included in the article's Creative Commons license and your intended use is not permitted by statutory regulation or exceeds the permitted use, you will need to obtain permission directly from the copyright holder. To view a copy of this license, visit http://creativecommons.org/licenses/by/4.0/.

(C) The Author(s) 2019 